SUBJECT AREAS:

TARGET IDENTIFICATION

MALARIA

Received

11 October 2013

Accepted

20 December 2013

Published

17 January 2014

Correspondence and requests for materials should be addressed to K.C.W. (kwilli4@luc. edu) or W.Z. (wzheng@mail.nih. gov)

* These authors contributed equally to this work.

\title{
Chemical signatures and new drug targets for gametocytocidal drug development
}

\author{
Wei Sun ${ }^{*}$, Takeshi Q. Tanaka ${ }^{2 *}$, Crystal T. Magle ${ }^{3}$, Wenwei Huang' ', Noel Southall', Ruili Huang', \\ Seameen J. Dehdashti' , John C. McKew' ${ }^{1}$ Kim C. Williamson ${ }^{2,3}$ \& Wei Zheng'
}

${ }^{1}$ National Center for Advancing Translational Sciences, National Institutes of Health, Bethesda, MD 20892, United States,
2Laboratory of Malaria and Vector Research, National Institute of Allergy and Infectious Diseases, National Institutes of Health,
Bethesda, MD 20892, United States, ${ }^{3}$ Department of Biology, Loyola University Chicago, Chicago, IL 60660, United States.

Control of parasite transmission is critical for the eradication of malaria. However, most antimalarial drugs are not active against $P$. falciparum gametocytes, responsible for the spread of malaria. Consequently, patients can remain infectious for weeks after the clearance of asexual parasites and clinical symptoms. Here we report the identification of 27 potent gametocytocidal compounds $\left(\mathrm{IC}_{50}<1 \mu \mathrm{M}\right)$ from screening 5,215 known drugs and compounds. All these compounds were active against three strains of gametocytes with different drug sensitivities and geographical origins, 3D7, HB3 and Dd2. Cheminformatic analysis revealed chemical signatures for $P$. falciparum sexual and asexual stages indicative of druggability and suggesting potential targets. Torin 2 , a top lead compound $\left(\mathrm{IC}_{50}=8 \mathrm{nM}\right.$ against gametocytes in vitro), completely blocked oocyst formation in a mouse model of transmission. These results provide critical new leads and potential targets to expand the repertoire of malaria transmission-blocking reagents.

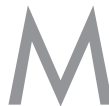
alaria cases and deaths have dropped 50\% in 29 countries since 2000 due to the combined effects of longlasting insecticidal bed nets, indoor residual spraying, and artemisinin-based combination therapies (ACTs) ${ }^{1}$. This success has raised hopes for malaria eradication and consequently stimulated interest in developing new reagents that block gametocyte transmission, such as novel and safe gametocytocidal drugs ${ }^{2}$. Previous drug development efforts have focused primarily on the asexual parasites that cause symptoms but not malaria transmission. To be transmitted from person to person via mosquitoes, the parasites must switch from asexual to sexual development and produce male and female gametocytes. Once gametocytes are taken up in a blood meal by a mosquito, fertilization is stimulated and the resulting zygote differentiates into a motile ookinete that migrates across the midgut epithelium and forms an oocyst. Over the course of the next 2 weeks, tens of thousands infectious sporozoites are generated and sequestered in the mosquito salivary glands until released into a vertebrate host for transmission during the next blood meal.

Sexual stage $P$. falciparum gametocytes have a lifespan of over 3 weeks and are not cleared effectively by current antimalarial agents, except primaquine $(\mathrm{PQ})^{3,4}$, which is not widely used because it causes hemolytic anemia in patients with glucose-6-phosphate dehydrogenase deficiency ${ }^{5}$. Consequently, treatment with current antimalarial drugs often results in asymptomatic carriers who remain infectious for weeks after the clearance of asexual parasites. Despite the risks of $\mathrm{PQ}$, its efficacy with artemisinin combination therapy (ACT) in reducing malaria transmission in the PQ-tolerant patients was recently demonstrated in test regions ${ }^{6}$. Other than PQ, the only other anti-gametocytocidal candidate being tested is methylene blue ${ }^{7}$. Thus, a new generation of antimalarial agents with potent activities against both sexual and asexual parasites is urgently needed for better therapeutic effect and eradication of malarial infection globally.

Due to the limited yield of gametocytes prepared from in vitro culture and assay sensitivity, high throughput gametocyte viability assays have only recently been developed ${ }^{2,8,9}$. We have screened 5,215 known compounds using the alamarBlue gametocyte viability assay and identified 27 novel gametocytocidal compounds. Because most of these compounds are approved drugs, a cheminformatic analysis of the screening data generated a profile of gametocytocidal compounds that were compared with those active against asexual parasites. These chemical signatures of known drugs suggest stage specific pathways as well as potential drug targets for both sexual (gametocytes) and asexual stages of the parasites including heat shock protein 90 (HSP90), aurora kinase (ARK1) and phosphatidylinositol 3-kinase (PI3K). A top lead compound, Torin 2, was confirmed with potent 
activities against both gametocytes and asexual parasites. Potential protein targets for this compound were also identified using affinity precipitation and drug affinity responsive target stability (DARTS $)^{10}$. Furthermore, oocyst formation in mosquitoes was completely blocked by Torin 2 in a mouse model of transmission. Therefore, the identified lead gametocytocidal compounds as well as potential new drug targets and pathways essential for gametocyte development provide new directions for the design of the next generation antimalarial agents.

\section{Results}

Identification of 27 gametocytocidal compounds. $P$. falciparum strain 3D7 gametocytes were screened against 5,215 compounds at four concentrations ranging from 0.37 to $46 \mu \mathrm{M}$ using an alamarBlue viability assay ${ }^{9,11}$. These compounds include 4,265 approved human or animal drugs ${ }^{12}, 400$ from the Malaria Box library that are active against $P$. falciparum strain 3D7 asexual parasites in $v$ itro $^{13}$, and 550 from an internal collection of kinase inhibitors ${ }^{14}$. A total of 27 novel active gametocytocidal compounds were identified and confirmed with $\mathrm{IC}_{50}$ values $\leq 1 \mu \mathrm{M}$ against gametocytes. Among these confirmed compounds, 21 had more than 10 -fold selectivity against gametocytes over the mammalian cell line HepG2 (Table 1 and SI Table 1). Gametocytocidal activities of these 27 compounds have not been previously reported elsewhere. NSC174938, Torin 2, NVP-AUY922, maduramicin, and narasin were the most potent compounds against gametocytes with $\mathrm{IC}_{50}$ values ranging from 3 to $50 \mathrm{nM}$ (Fig. 1A, 1B, and Table 1). Two compounds in the malaria box reduced gametocyte viability by $>75 \%$ and had an IC $_{50}$ of $<1 \mu \mathrm{M}$, MMV019406 and MMV666125 (SI Table 2). Additionally, PQ and 7 other compounds with known gametocytocidal activity were present in the compound collection and were all identified in the screen (Table 1), validating the effectiveness of this screening method. The $\mathrm{IC}_{50} \mathrm{~s}$ of the endoperoxides were similar to those previously reported for gametocyte stages IV-V ${ }^{15}$, but lower than the $\mathrm{IC}_{50}$ s recently reported using an ATPase assay ${ }^{16}$. The reason for this difference is unknown, although the length of drug exposure and gametocyte purification methods varied among these assays.

Table 1 | Compounds with potent activity against $P$. falciparum 3D7 gametocytes

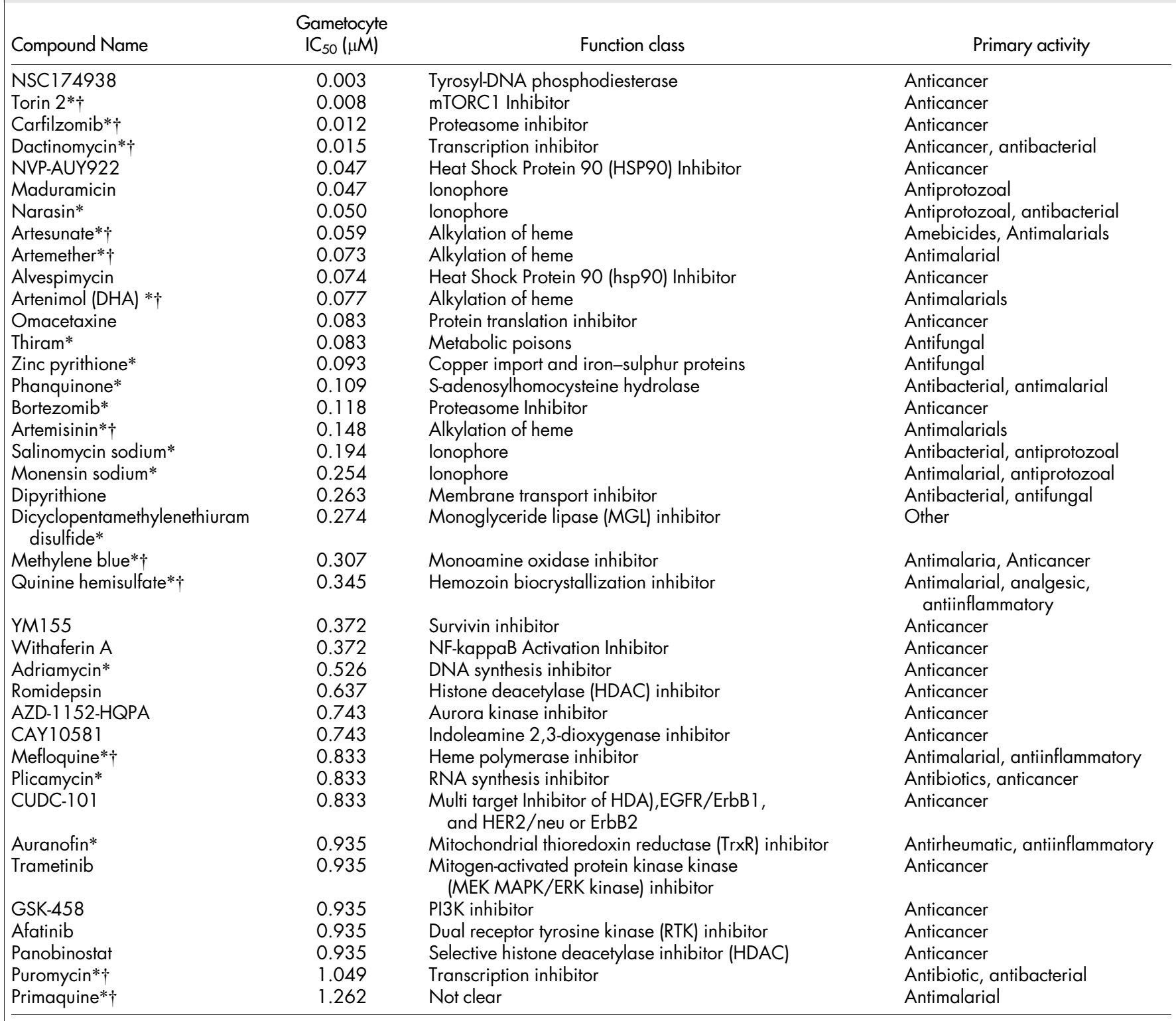

Note: mean $\mathrm{I} \mathrm{C}_{50}$, mean half-maximum inhibitory concentrations determined from at leas 3 independent experiments against $P$. falciparum $3 \mathrm{D} 7$ gametocyte; $*$ indicates compounds with previously reported activities against asexual parasites. ${ }^{\dagger}$ means compounds with previously reported activities against gametocytes (references are in supplementary information). 

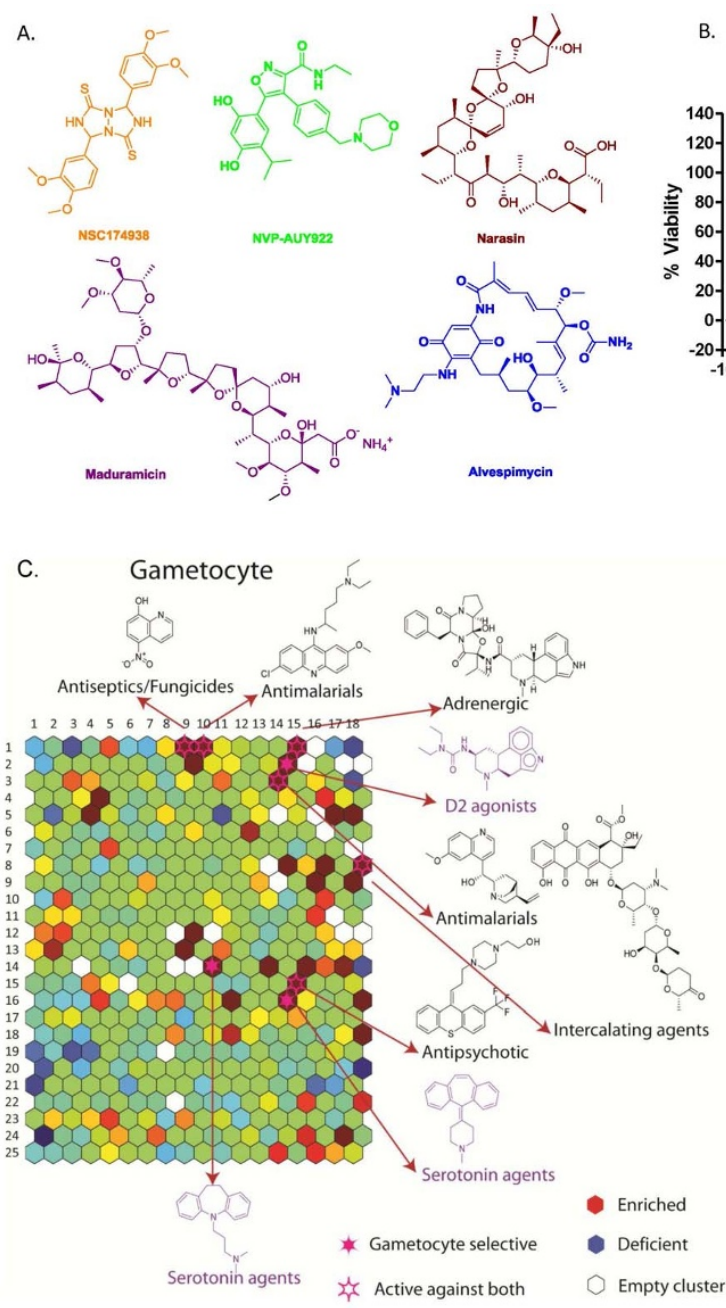

B.

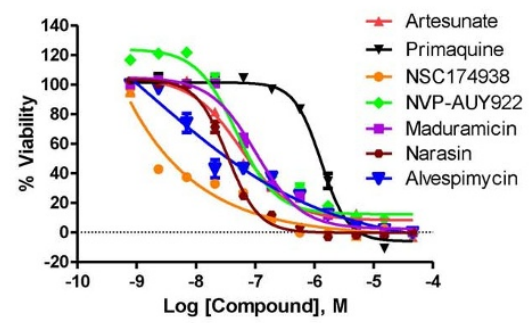

Asexual

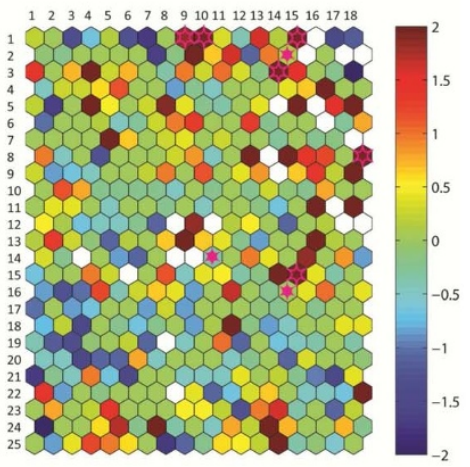

D.

Drug Classes

Target Proteins/Pathways

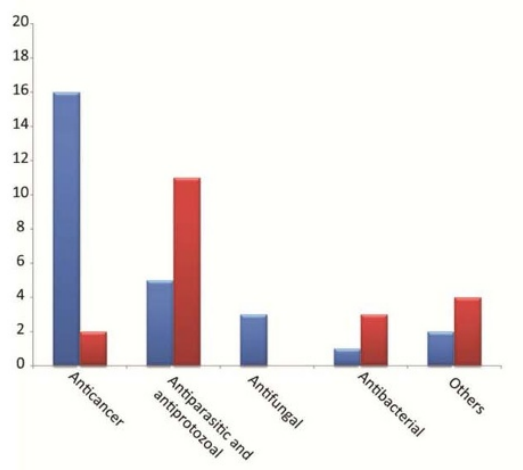

27 Newly identified compounds 27 Newly identified compounds

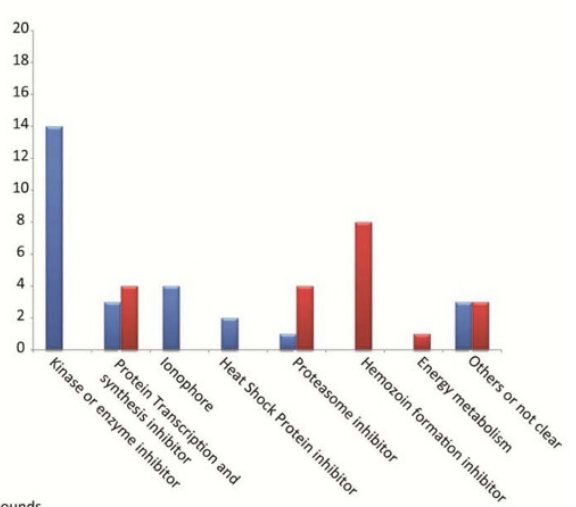

Figure $1 \mid$ Cheminformatic summary of drug repurposing screen against $P$. falciparum $3 D 7$ gametocytes. (A) Structures of top representative gametocytocidal compounds. (B) Concentration-response curves of selected lead compounds (NSC174938, NVP-AUY922, maduramicin, narasin, alvespimycin, primaquine and artesunate) determined in the gametocyte viability assay. (C) Structure clustering of compound activity across the compounds screened. In the heat maps, each hexagon represents a cluster of compounds with structural similarity. Red colored clusters represent structures enriched in compounds active against the parasites as measured by a Fisher's exact test. Blue colored clusters represent structures with minimal active compounds. Coloring is scaled by the negative $\log 10$ of the P-values. Darker in red or blue color indicates a higher level of enrichment or absence of active compounds in each structure cluster. Compound structures show the examples of known drug groups active against both gametocytes and asexual parasites (red hexagons in both heat maps) or selectively active against gametocytes over asexual parasites (red hexagons in the gametocyte map and greenish or blue in the asexual map with structures and annotations highlighted in purple). (D) Distribution of known drug indications and targets/ pathways of 27 newly identified gametocytocidal compounds compared to 20 previously reported gametocytocidal compounds. Left panel: number of active compounds in each drug class. If a compound has more than one indication, it is counted once by the following order: antiparasitic and antiprotozoal, antifungal, antibacterial, anticancer or others. Right panel: number of active compounds in each known drug targets/pathways. 
Cheminformatic analysis of gametocytocidal activity compared to activity against asexual parasites. In addition to the 27 potent compounds analyzed above, many others among the 5,215 compounds screened also exhibited gametocytocidal activity. Most of the compounds screened in this experiment had been previously profiled against the asexual stages of $P$. falciparum strain 3D7 and its clinical variants ${ }^{17,18}$. These two previous studies demonstrate the utility of profiling chemical genomic signatures of asexual parasites by screening the approved drug collection. To identify structural motifs that are selectively active against gametocytes over asexual parasites, we clustered these compounds based on their structural similarity (2,048-bit Daylight fingerprints; Daylight Chemical Information Systems, Inc., Laguna Niguel, CA) using the selforganizing map (SOM) algorithm ${ }^{19}$ (Fig. 1C). Each hexagon in the SOMs represents a cluster of structurally similar compounds, with neighboring hexagons containing more similar structures than distal hexagons and is designated by their row and column number. This analysis identified a number of compound classes that are active against both gametocytes and asexual parasites (Fig. 1C, hexagons colored red in both the gametocyte and the asexual SOMs), including DNA intercalating agents (8.18), certain classes of antipsychotic drugs (15.15) and adrenergic agents (1.15), as well as the groups containing the antimalarials, primaquine (1.10) and mefloquine (3.14) and classes of antiseptics and fungicides (1.9). We also found structural motifs that appear to be specific against gametocytes (SI Table 3 and Fig. 1C, hexagons colored red in the gametocyte SOM but greenish or blue in the asexual SOM), exemplified by a class of D2 agonists (2.14) and certain classes of serotonin agents (14.10 and 16.14). The analysis also confirmed the specificity of dihydrofolate reductase (DHFR) inhibitors against asexual stages of parasites ${ }^{20}$. Together, the results indicate the druggability of $P$. falciparum gametocytes by chemical motifs that exist in approved drugs as well as selectivity of these compounds against the gametocytes.

New targets for gametocytocidal drug development. Many active compounds identified by screening a known drug library cannot be directly used in patients because effective compound concentrations in plasma or tissue for the new indication cannot be achieved. However, compounds with known mechanism of action provide valuable information for identification of their targets. These compounds can be optimized, or new compounds can be developed against the newly identified drug targets. In this case, the selective compounds may be developed based on the significant differences between malarial and human proteins. Because most screened compounds are known drugs or pharmacologically active compounds, we further analyzed the parasite stage specific compounds to identify potential new targets for gametocytocidal drug development and pathways potentially required for gametocyte development. Compound activities against $P$. falciparum gametocytes were also compared with those obtained in previous screens against asexual parasites. Based on compound selectivity, three groups of compounds were identified: (1) those selective against gametocytes, (2) those selective against asexual parasites, and (3) those active against both stages of the parasites (SI Table 4). Each group was tested for enrichment with known targets and/or therapeutic indications (significantly enriched, Fisher's exact test; $\mathrm{p}<0.05)$. We found that drugs acting on cholinergic receptors (muscarinic 1 and 2), 5-Hydroxytryptamine (serotonin) receptor $2 \mathrm{~A}$ and solute carrier family 6 are gametocyteselective. Interestingly, no close homologues for these proteins have been found in P. falciparum. However, a tricyclic structure is a common theme present in these active compounds, indicating that this structure may bind well to a putative gametocyte-specific protein. In contrast, drugs acting on DHFR are selective for asexual parasites, which is consistent with a previous report ${ }^{21}$. And compounds active against topoisomerases and potassium voltagegated channels are effective on both gametocytes and asexual parasites (SI Table 4).

Additionally, drug indication analysis of the top 27 potent gametocytocidal compounds revealed that $59 \%$ are known anticancer agents, while only $19 \%$ are previously established antiparasitic/antiprotozoal agents. The remaining $22 \%$ are categorized as antibacterials, antifungals or others (Fig. 1D). Furthermore, analysis of compounds with known drug targets revealed that $52 \%$ of newly identified target proteins are kinases/other enzymes including $\mathrm{ARK} 1^{22}$, and histone deacetylase (HDAC) $)^{23}, 11 \%$ are proteins involved in protein transcription/synthesis, 7\% are heat shock proteins, $4 \%$ are proteasome components and $11 \%$ have other functions. The remaining $15 \%$ of the compounds are classified as ionophores (Fig. 1D). However, annotated P. falciparum homologues could only be found for seven of the target enzymes predicted by prior analysis of the approved drugs on mammalian cells (SI Table 5). By comparison, $55 \%$ of the 20 previously identified gametocytocidal compounds (Table 1 and SI Table 6), except $\mathrm{PQ}^{24}$, are more potent against asexual parasites than gametocytes, which accounts for the ineffectiveness in blocking malaria transmission by routine antimalarial treatment ${ }^{25,26}$. Additionally, $40 \%$ of the 20 previously identified gametocytocidal compounds including the four artemisinin derivatives tested have been reported to affect hemozoin formation, 20\% target the proteasome, $20 \%$ affect DNA transcription, $5 \%$ are related to energy metabolism, and $15 \%$ have other functions. Together, we have identified several groups of new gametocyte targets, providing alternative approaches for future antimalarial drug development.

Profiles of gametocytocidal compounds against drug resistant strains. Drug resistance is also a critical challenge for malaria treatment and eradication that has not been examined in gametocytes, though it has been extensively studied for the asexual parasites ${ }^{27,28}$. To evaluate whether existing antimalarial agents and newly identified gametocytocidal compounds are effective against well characterized drug resistant strains, we determined the gametocytocidal activities of 52 selected compounds, including 27 newly identified compounds and 25 known antimalarial agents, against gametocytes of $P$. falciparum strains Dd2 and HB3 in the alamarBlue viability assay. In contrast to $3 \mathrm{D} 7$, asexual $\mathrm{Dd} 2$ parasites are resistant to chloroquine, mefloquine and pyrimethamine while asexual HB3 parasites are resistant to pyrimethamine but not chloroquine or mefloquine ${ }^{29}$. Most of 52 compounds showed 5fold or less differences in potency between these two parasite strains compared to the drug sensitive stain 3D7 (Fig. 2A, 2B and SI Table 7). Compared to the drug sensitive stain 3D7, chloroquine's potency against Dd2 gametocytes was reduced 3.7-fold while it was 10 -fold less potent against Dd2 asexual parasites ${ }^{29}$. Methylene blue was moderately more active against $3 \mathrm{D} 7$ gametocytes $\left(\mathrm{IC}_{50}=\right.$ $0.307 \mu \mathrm{M})$ than those of HB3 $\left(\mathrm{IC}_{50}=0.935 \mu \mathrm{M}\right)$ and $\mathrm{Dd} 2\left(\mathrm{IC}_{50}\right.$ $=0.526 \mu \mathrm{M})(\mathrm{SI}$ Table 7$)$. PQ showed similar potencies against gametocytes from these three strains with $\mathrm{IC}_{50}$ values of $1.26,0.68$, and $1.08 \mu \mathrm{M}$ against 3D7, HB3, and Dd2, respectively (Fig. 2A).

Interestingly, several of these newly identified gametocytocidal compounds exhibited similar or higher activities in these two asexual drug resistant strains compared to the drug sensitive 3D7 strain. For example, CUDC-101, a multi-target anticancer drug candidate ${ }^{30}$, was 5.5 -fold more potent against $\mathrm{HB} 3\left(\mathrm{IC}_{50}=0.152 \mu \mathrm{M}\right)$ compared to 3D7 $\left(\mathrm{IC}_{50}=0.833 \mu \mathrm{M}\right)$ (Fig. 2A). Additionally, panobinostat, a histone deacetylase inhibitor, was also 6.3 to 7.9 times more potent against $\mathrm{Dd} 2\left(\mathrm{IC}_{50}=0.148 \mu \mathrm{M}\right)$ and $\mathrm{HB} 3\left(\mathrm{IC}_{50}=0.118 \mu \mathrm{M}\right)$ compared to $3 \mathrm{D} 7\left(\mathrm{IC}_{50}=0.935 \mu \mathrm{M}\right)($ Fig. $2 \mathrm{~A})$. These results suggest that these newly identified gametocytocidal compounds could also be effective against a range of asexual drug resistant isolates.

Activities of Torin 2 against gametocytes and asexual parasites in vitro. Torin 2 , a known $\mathrm{mTOR}$ inhibitor ${ }^{31}$, was one of the most 

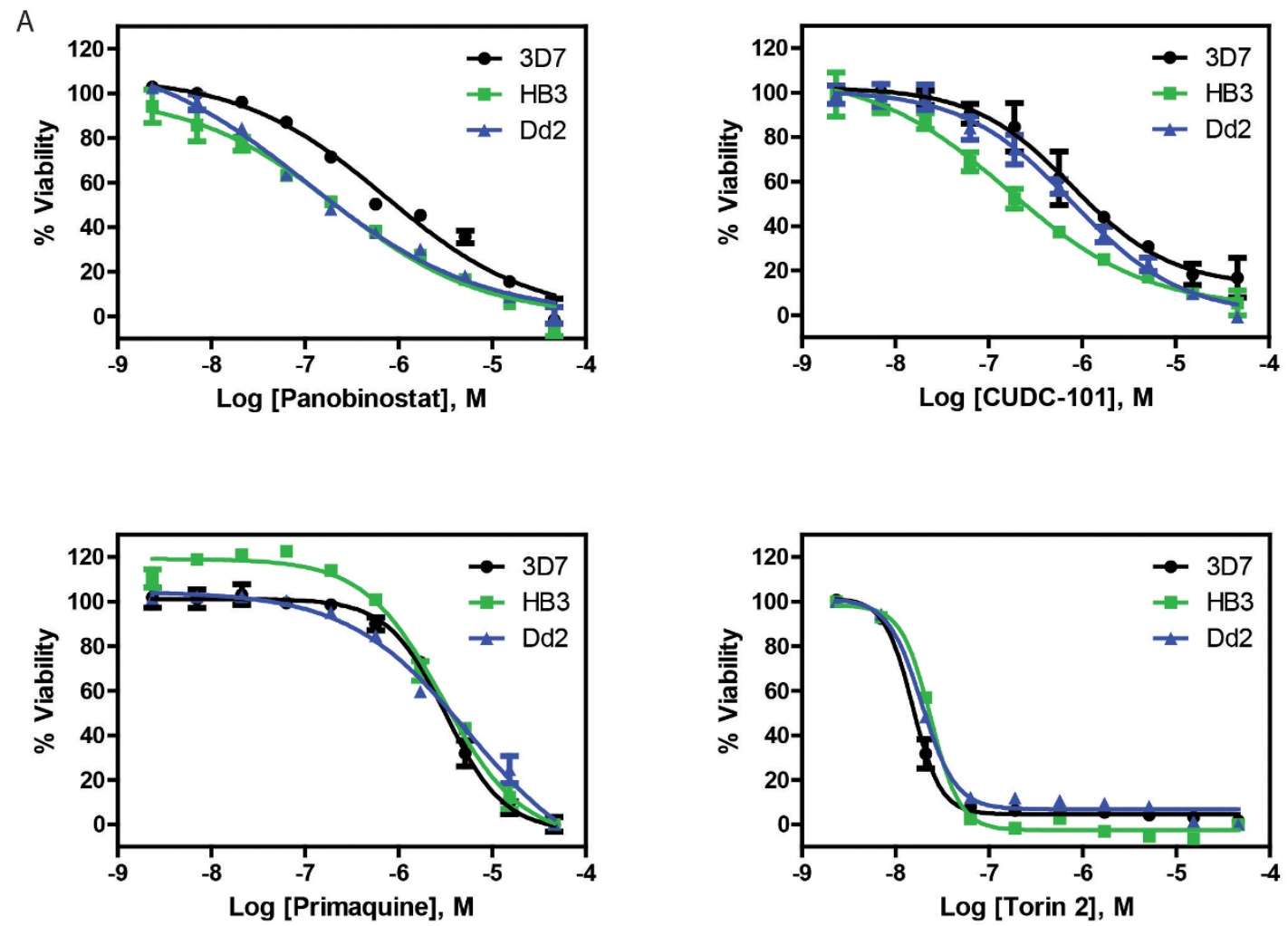

B

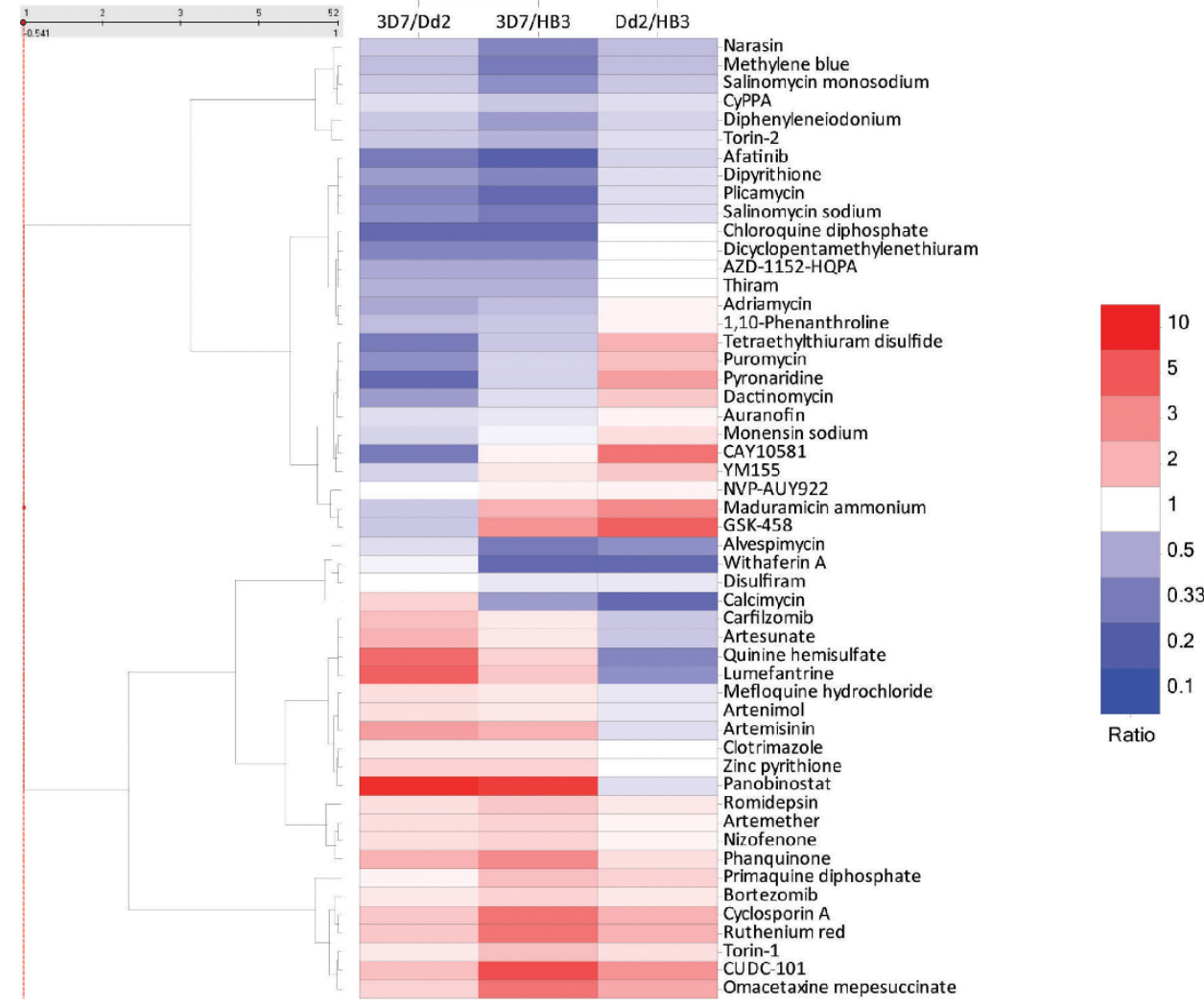

Figure $2 \mid$ Profiling of gametocytocidal compounds against $P$. falciparum gametocytes of drug sensitive strain 3D7 and drug resistant strains $\mathrm{HB} 3$ and Dd2. Asexual Dd2 parasites are resistant to chloroquine, mefloquine, and pyrimethamine while HB3 is resistant to pyrimethamine but not chloroquine or mefloquine. (A) Concentration-response curves of two representative strain selective compounds panobinostat and CUDC-101 (with potencies greater than 5-fold to HB3/Dd2 strains) in comparison with strain nonselective compounds primaquine and Torin 2. (B) Comparison of compound potencies across three strains. For example, a compound colored in white in 3D7/Dd2 group indicates equal potencies in both strains, whereas the one in dark red indicates that the compound is more potent against $\mathrm{Dd} 2$ and the one in dark blue means that the compound is more potent against 3D7. Compounds in the heat map are hierarchically clustered by their $\mathrm{IC}_{50}$ ratio profile across the three strains. 
potent new gametocytocidal compounds $\left(\mathrm{IC}_{50}=8 \mathrm{nM}\right.$, Table 1$)$. In contrast, its structural analog, Torin 1 , was 200 -fold less potent $\left(\mathrm{IC}_{50}\right.$ $=1.6 \mu \mathrm{M}$, Fig. $3 \mathrm{~B}$ ), regardless of their similar potencies on mTOR $\left(\mathrm{IC}_{50} \text { values of } 5.4 \text { and } 2.1 \mathrm{nM} \text {, respectively }\right)^{32}$. The difference in gametocytocidal activity between the two compounds was confirmed using the traditional gametocyte viability assay, optical microscopy of Giemsa stained smears (SI Fig. 2A). Both Torin 1 and 2 share the same core structure, benzo[h] $[1,6]$ naphthyridin-2 $(1 \mathrm{H})$ one, but the 2 -aminopyridine in Torin 2 is replaced with a quinolone in Torin 1 (Fig. 3A). The 200-fold difference in potencies against $P$. falciparum gametocytes suggests that Torin 2 and Torin 1 may act on a target or targets other than mTOR, consistent with the lack of mTOR homolog in P. falciparum ${ }^{33}$.

To further characterize the utility of Torin 2 as an antimalarial drug, its in vitro activity against asexual blood stage parasites and HepG2 cells was also evaluated. Torin 2 exhibited an $\mathrm{IC}_{50}$ of $2.75 \mathrm{nM}$ (Fig. 3C) against asexual parasites, while Torin 1 had an $\mathrm{IC}_{50}$ of $215 \mathrm{nM}$ (SI Fig. 2B), making Torin 1 78-times less potent than Torin 2. Notably, both compounds were more potent against the asexual parasites than gametocytes. Additionally, Torin-2 toxicity in mammalian cells was assessed using HepG2 cells (Fig. 3C and SI Table 1). Torin 2 exhibited only partial cytotoxicity at the highest tested concentration $(46 \mu \mathrm{M})$, indicative of greater than 1,000 -fold selectivity against the parasites over the mammalian cells (SI Table 1). Taken together, the results demonstrate the similar low nanomolar potencies of Torin 2 against both sexual and asexual stages of $P$. falciparum, as well as its high selectivity against $P$. falciparum parasites over mammalian cells.

Efficacy of Torin 2 on gametocyte transmission from vertebrate host to mosquitoes in a mouse model. The transmission of Plasmodium berghei ANKA $(\mathrm{Pb})$ from infected mice to Anopheles stephensi mosquitoes was examined to investigate the in vivo efficacy of Torin 2. The experiment was designed to accommodate developmental differences between $P$. berghei and P. falciparum. In contrast to $P$. falciparum, which requires $48 \mathrm{hrs}$ to complete its asexual life cycle and 10-12 days to complete sexual differentiation, $P$. berghei completes asexual replication and sexual differentiation cycles in $24 \mathrm{hrs}$. $P b$-infected mice were assessed for exflagellation to monitor the presence of $P$. berghei gametocytes, and drug or vehicle control were administered when $>1$ exflagellation center per two $400 \times$ field were observed. Some mice were given two doses of drug three hrs apart to increase exposure time, but on a short enough time scale to prevent a concomitant decrease in asexual parasite from affecting the next cycle of gametocytes. This dosing schedule was based on the transmission experiments done with primaquine ${ }^{34}$. The mosquito feed assay was performed $1.5 \mathrm{hrs}$ after the final drug treatment as described in the Methods (Fig. 4A). Exflagellation was also monitored just prior to mosquito feed, and no difference was observed between the drug- and vehicletreated mice (data not shown). In contrast, oocyst production in the fed mosquitoes, which was used as an indicator of malaria transmission, was completely blocked by treatment with two $4 \mathrm{mg} / \mathrm{kg}$ doses of Torin 2 in 3 independent experiments while 73\% of the mosquitoes fed on control mice had $>45$ oocysts/midgut (Fig. 4B, p $<0.001$, Students T-test). To further evaluate the dose dependence, we tested single 2 or $4 \mathrm{mg} / \mathrm{kg}$ doses of Torin 2 in the same mouse model (Fig. 4C). A single dose of $2 \mathrm{mg} / \mathrm{kg}$ of Torin 2 significantly reduced oocyst production, while a single $4 \mathrm{mg} / \mathrm{kg}$ dose almost completely eliminated it. These results clearly demonstrate the ability of Torin 2 to prevent oocyst formation in mosquitoes in a dose dependent manner.
A<smiles>Nc1ccc(-c2ccc3ncc4ccc(=O)n(-c5cccc(C(F)(F)F)c5)c4c3c2)cn1</smiles>

Torin $2\left(\mathrm{IC}_{50}=8 \mathrm{nM}\right)$<smiles>CCC(=O)N1CCN(c2ccc(-n3c(=O)ccc4cnc5ccc(-c6cnc7ccccc7c6)cc5c43)cc2C(F)(F)F)CC1</smiles>

Torin $1\left(\mathrm{IC}_{50}=1600 \mathrm{nM}\right)$<smiles>CCC(=O)N1CCN(c2ccc(-n3c(=O)ccc4cnc5ccc(-c6ccc(N)nc6)cc5c43)cc2C(F)(F)F)CC1</smiles>

WWH030 $\left(\mathrm{IC}_{50}=9 \mathrm{nM}\right)$
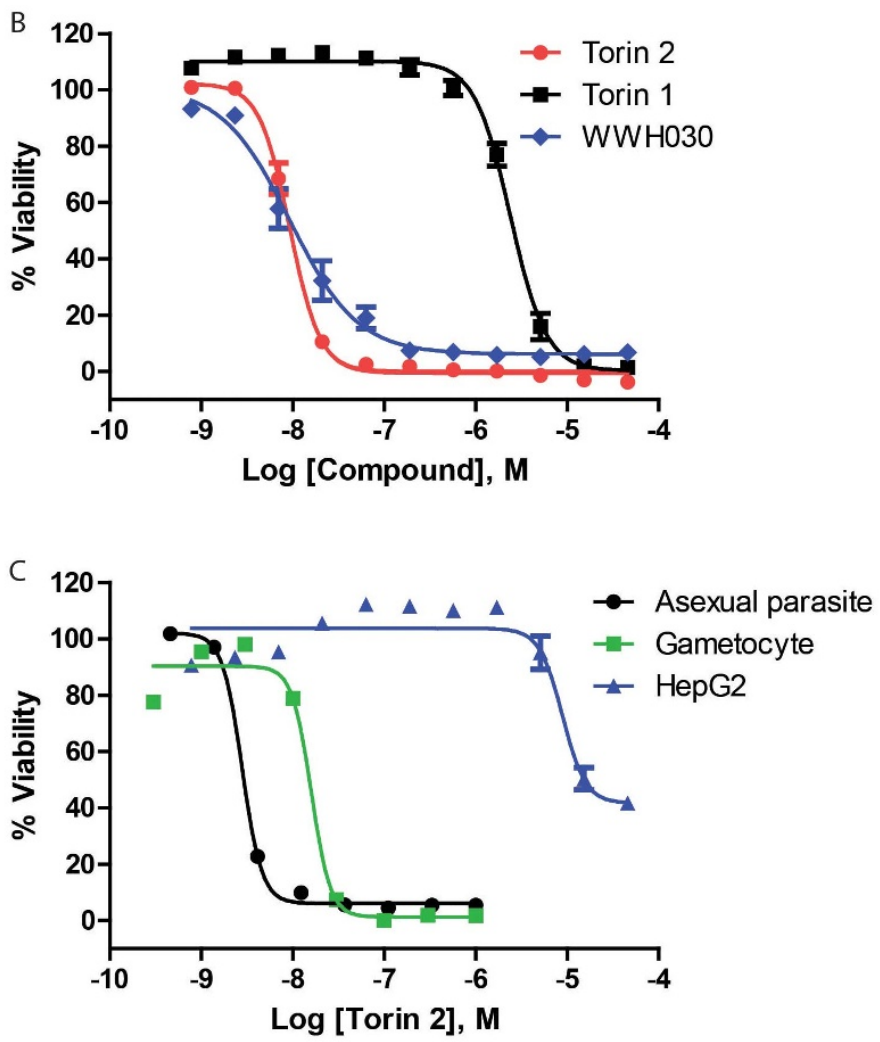

Figure 3 Structures and activities of Torin 2 and its analogs. (A) Chemical structures of Torin 2, Torin 1 and WWH030. (B) Concentration-response curves of Torin 2, Torin 1 and WWH030 against gametocytes measured in the high throughout viability assay. Torin 1 was much weaker in potency compared to Torin 2. (C) Concentration-response curves of Torin 2 determined in the optic microscopic gametocyte assay, in the asexual parasite SYBR green assays and in the mammalian HepG2 cell cytotoxicity assay. Torin 2 exhibited a great selectivity to malaria parasites over the mammalian cells. 
A

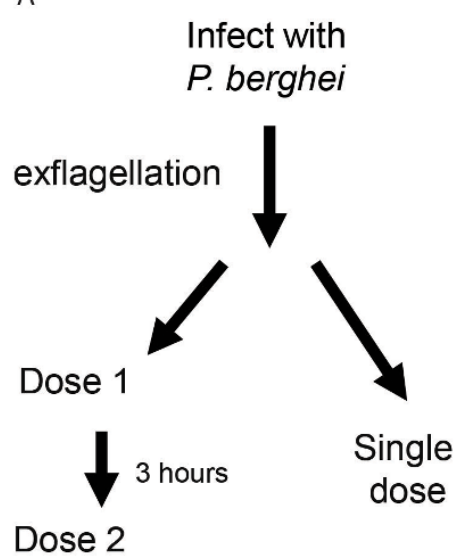

Dose 2

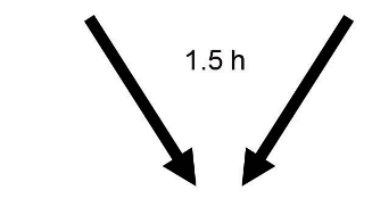

Feed mice to $A$. stephensi

7-10 Days

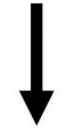

Count oocysts in A. stephensi
B
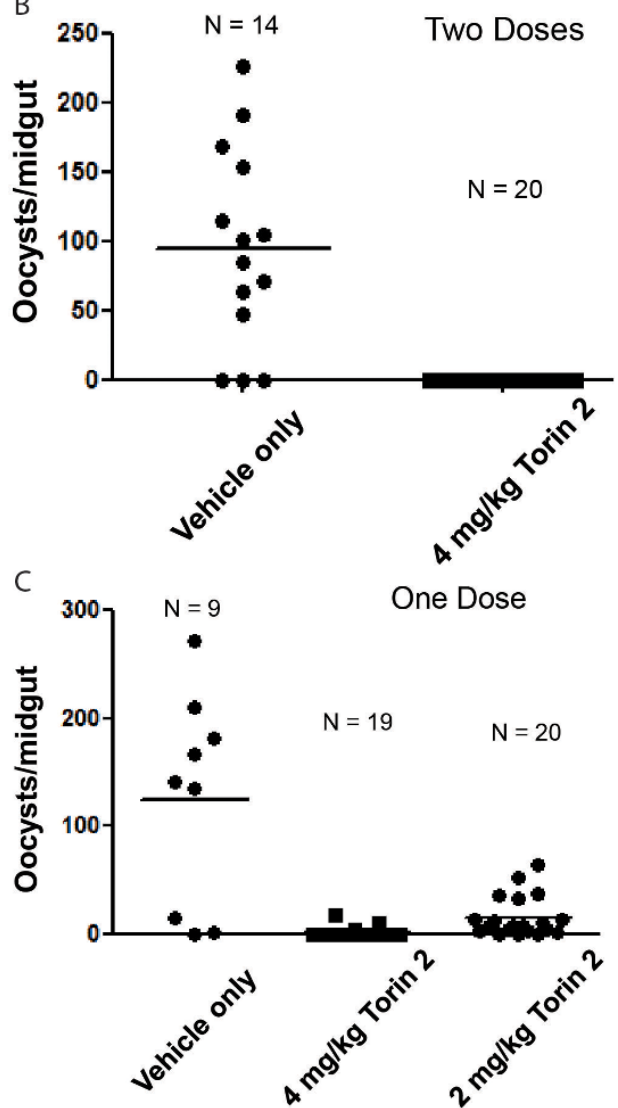

Figure 4 In vivo efficacy of Torin 2 in a mouse model of transmission. (A) Schematic process of mouse-mosquito transmission using $P$. berghei and $A$. stephensi. Mice infected with P. berghei were treated with drug or vehicle alone. $1.5 \mathrm{hr}$ after drug treatment, A. stephensi was allowed to feed on the infected mice to test parasite transmission. A. stephensi were maintained for 10 days to allow oocyst development, and the numbers of oocysts per mosquito midgut were recorded. (B) Two doses of $4 \mathrm{mg} / \mathrm{kg}$ Torin 2 given $3 \mathrm{hrs}$ apart completely prevented oocyst formation in mosquitoes that fed on the infected mice 1.5 hrs post drug treatment. A representative experiment is shown ( $\mathrm{p}<0.001$, Student's T-test). (C) A single dose of $2 \mathrm{mg} / \mathrm{kg}$ Torin 2 significantly reduced oocyst production in contrast to nearly complete elimination of oocyst production by a single dose of $4 \mathrm{mg} / \mathrm{kg}$ Torin 2 (p $<0.001$, one way ANOVA with Tukey's post-hoc test).

Identification of potential molecular targets of Torin 2. The lack of an mTOR homologue in $P$. falciparum ${ }^{33}$ and the significant difference in the potencies of Torin 1 and Torin 2 against the parasites suggest the presence of distinct targets in the parasites. We hypothesized that Torin 2 selectively interacts with an unknown $P$. falciparum protein (or proteins) that has a weaker binding affinity to Torin 1 . To develop a probe for an affinity based pull-down experiment, a Torin derivative, WWH030, was synthesized, and the importance of the ortho-peperzine-amide on the (trifluoromethyl)-benzene of Torin 2 for its gametocytocidal activity was determined (Fig. 3A). The new derivative had an $\mathrm{IC}_{50}$ of $9 \mathrm{nM}$, similar to that of Torin 2 in the gametocyte assay (Fig. $3 \mathrm{~B}$ ). This result indicates that the ortho-peperzine-amide group on Torin 2 can be modified without a significant effect on its gametocytocidal activity. Therefore, T2M was synthesized as an affinity resin for the pull-down experiment for identification of Torin 2 interacting proteins in $P$. falciparum gametocyte lysates (Fig. 5A). A negative control resin, T1M, was similarly synthesized with a close analog of Torin 1 (Fig. 5A).

The proteins precipitated from gametocyte lysate by T2M but not $\mathrm{T} 1 \mathrm{M}$ were identified by mass spectrometric analysis (Fig. $5 \mathrm{~B})^{35}$. The proteomics data revealed a total of 31 proteins selectively enriched by T2M (SI Table 8). In parallel to the probe-protein precipitation experiment, we also carried out a DARTS experiment ${ }^{10}$ to identify Torin 2 binding proteins by limited protease digestion of Torin 2treated gametocyte lysates. Following treatment with either Torin 2 or the negative control Torin 1, gametocyte lysates were partially digested with pronase and size fractionated by SDS-PAGE (Fig. 5C). Four significant protein bands were enriched in the Torin 2-treated sample compared to the Torin 1-treated sample and analyzed by mass spectrometry. After comparing the results from the affinity precipitation experiment, we found that phosphoribosylpyrophosphate synthetase (PF3D7_1325100, ribosephosphate diphosphokinase), aspartate carbamoyltransferase (PF3D7_ 1344800, ATCase), and a putative transporter (PF3D7_0914700) were identified by both experiments (Table 2 ). Thus, these three gametocyte proteins are potential drug targets for Torin 2 and will need to be further confirmed by enzyme assays and binding assays using recombinant P. falciaprum proteins.

\section{Discussion}

Taking advantage of the miniaturized gametocyte viability assay, we have screened 5,215 approved drugs and known compounds to profile the gametocytocidal activity of these compounds. Among the 27 potent gametocytocidal compounds identified, 16 are completely new to the malaria research community and the other 11 have known activity against asexual parasites (Table 1). The chemical informatics analysis of these compounds in combination with their known mechanisms of action have revealed a set of target proteins such as HSP90, HDAC, ARK1, PI3K, S-adenosylhomocysteine hydrolase $(\mathrm{SAHH})$, and thioredoxin reductase that are crucial for the growth and survival of malaria. Thus, these newly identified protein targets 
A<smiles>[R]Oc1ccc(OCCOCCOCCOCCOCc2ccccc2)cc1</smiles>

\section{.}
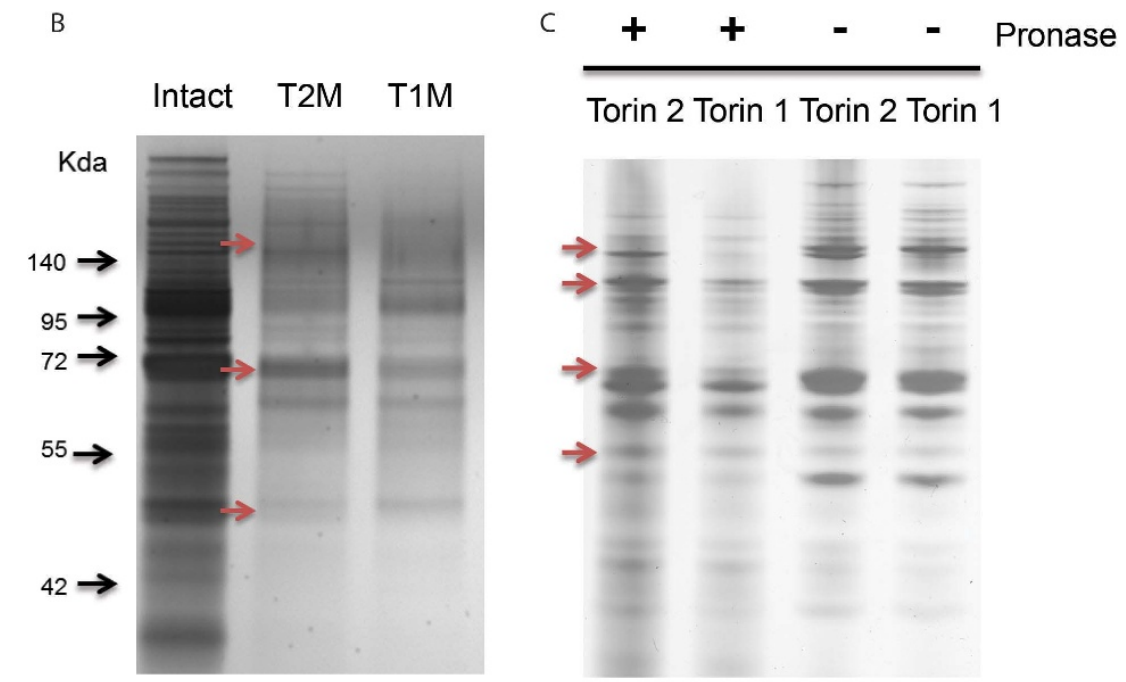

Figure 5 Target identification for potential Torin 2 interacting proteins by affinity precipitation and drug affinity responsive target stability (DARTS). (A) Chemical structures of Torin 2 matrix (T2M) and Torin 1 matrix (T1M) for the affinity precipitation (pull-down) experiment. Torin 1 matrix probe was used as a negative control. (B) Protein bands identified by the affinity precipitation experiment using T2M in comparison with T1M negative control. P. falciparum 3D7 gametocyte lysates were incubated with the affinity probes at $4{ }^{\circ} \mathrm{C}$ for 2 hrs. Bound proteins were eluted, resolved on a $10 \%$ Bis-Tris gel, and visualized with silver staining. The arrows indicated protein bands (selective to T2M probe) were processed for mass spectrum analysis. (C) Protein bands identified by the DARTS experiment. P. falciparum 3D7 gametocyte lysates were treated with either Torin 2 (lane 1 and 3 ) or Torin 1 (lane 2 and 4) at a concentration of $600 \mathrm{nM}$ for $1 \mathrm{hr}$. Each sample was split into two aliquots for proteolysis with (lane 1 and 2 ) or without (lane 3 and 4) pronase. The partially digested proteins were separated by $10 \%$ Bis-Tri gel and visualized with silver staining. The Torin 2 specific protein bands indicated by arrows were further processed for mass spectrum analysis.

provide a new direction for the study of essential signaling pathways in the parasites and the development of antimalarial agents against gametocytes.

Structurally similar compounds in the same chemical cluster often share a similar mode of action ${ }^{36}$. This approach is especially useful for phenotypic drug screens where specific target information is unavailable. Our cheminformatic analysis of compound activities obtained in this study and those previously reported for asexual parasites revealed both shared and distinct chemical signatures against gametocytes and asexual parasites (Fig. 1C). The analysis revealed several core templates (e.g. quinine and quinacrine types of antimalarial agents, anthracyclines, and dihydroergotamine type of adrenergic agents) that are active on both stages of the parasites. Dibenzazepine class of serotonin agents are a structure class that is specifically active in gametocyte stage, indicative of potential targets selectively expressed in gametocytes. The activities of antimalarial quinine analogs and anthracycline derivatives against both asexual parasites and gametocytes have been reported previously ${ }^{37,38}$, validating the utility of this analysis method. Dihydroergotamine types of adrenergic agents are a new cluster of compounds active against both

Table 2 | Three potential protein targets in gametocytes for Torin 2

\begin{tabular}{|c|c|c|c|c|c|c|c|}
\hline Name of protein & Accession Number & $\begin{array}{l}\text { Molecular } \\
\text { Weight }\end{array}$ & \multicolumn{2}{|c|}{ Pull-down } & \multicolumn{2}{|c|}{ DARTS } & Function \\
\hline $\begin{array}{l}\text { Phosphoribosyl pyrophosphate } \\
\text { synthetase (Ribose-phosphate } \\
\text { diphosphokinase) }\end{array}$ & PF3D7_1325100 & $49 \mathrm{kDa}$ & 4 & 0 & 6 & 0 & $\begin{array}{l}\text { Biosynthesis of purine, } \\
\text { pyrimidine and pyridine } \\
\text { nucleotides }\end{array}$ \\
\hline $\begin{array}{l}\text { Aspartate carbamoyltransferase } \\
\text { (ATCase) }\end{array}$ & PF3D7_1344800 & $43 \mathrm{kDa}$ & 2 & 0 & 4 & 0 & Biosynthesis of pyrimidine \\
\hline
\end{tabular}

Note: Protein bands in both positive (Torin 2 affinity precipitation) and negative (Torin 1 affinity precipitation) samples were destained, reduced, and digested for mass spectrum analysis. The mass spectrum data were analyzed by SEQUEST using plasmoDB genomic database. In DARTS assays, upon pronase treatment, protein bands in positive (Torin 2 protected) and negative (Torin 1 protected) samples were processed similar as in above affinity precipitation methods. Phosphoribosyl pyrophosphate synthetase, aspartate carbamoyltransferase, and transporter are the three proteins identified by both pull-down and DARTS. 
asexual parasites and gametocytes, whose activities were previously shown only against $P$. falciparum asexual parasites ${ }^{18}$. These observations indicate that the structurally similar compounds in the same clusters may share common modes of actions.

Kinases and other enzymes make up the largest percentage of potential protein targets identified from this study for gametocytocidal drug development. $48 \%$ of the active compounds in the previous screen against $P$. falciparum asexual parasites targeted kinases ${ }^{40}$, reflecting the abundance of these compounds in the library. Less than 100 kinases have been identified in P. falciparum, compared to over 450 kinases in human ${ }^{39}$. Our new data in combination with the data from previous reports for asexual parasites ${ }^{40,41}$ indicate that malaria kinases are a group of potential targets for development of new antimalarial agents that control both sexual and asexual parasites.

Additionally, two anticancer agents (NVP-AUY922 and alvespimycin), two ionophores (maduramicin and narasin) and the mTOR inhibitor Torin 2 exhibited potent activities against gametocytes of all three tested $P$. falciparum strains including two previously reported asexual drug resistant ones. NVP-AUY922 and alvespimycin are both potent inhibitors of HSP $90^{42,43}$. Currently NVP-AUY922 is being used as an experimental drug in late human clinical trials for the treatment of metastatic non-small cell lung cancer ${ }^{44}$ and alvespimycin has been tested in patients with advanced solid tumors ${ }^{45}$. Alvespimysin is a derivative of geldanamycin, a natural product that was known to kill asexual parasites, but its effect on malaria transmission was not tested ${ }^{46}$. Heat shock proteins have been extensively studied in $P$. falciparum and are predicted to be antimalarial targets, but their effects on gametocytes have not been reported before ${ }^{46-48}$. There are four $P$. falciparum HSP90 homologs including Pf3D7_ 708400, Pf3D7_1443900, Pf3D7_118200, and HSP90b1 P3D7_ 1222300 which share 33 to $68 \%$ homology with human HSP90s ${ }^{49}$. Pf3D7_708400 and Pf3D7_118200 are expressed throughout parasite development including stage $\mathrm{V}$ gametocytes, indicative of potential targets for antimalarial agents against multi-stage parasites.

Torin 2 is another strong candidate for further development. It effectively kills both asexual parasites and gametocytes of all three $P$. falciparum strains tested with similar potencies including the asexual drug resistant strains, $\mathrm{HB} 3$ and $\mathrm{Dd} 2{ }^{29}$. It also completely blocked $\mathrm{Pb}$ oocyst production in the mouse-mosquito transmission model and showed more than 1,000-fold selectivity for the parasites over mammalian cells. This compound has been reported as a potent second generation ATP-competitive inhibitor of $\mathrm{mTOR}^{32}$ with good oral bioavailability (51\%). In mice, its concentrations in plasma, liver and lung maintained strong mTOR inhibition for 6 hours after a single dose of $20 \mathrm{mg} / \mathrm{kg}^{32}$. The antitumor activity of Torin 2 was observed in KRAS-driven lung tumors in combination with mitogen-activated protein/extracellular signal-regulated kinase (MEK) inhibitor AZD6244 ${ }^{31}$. The mammalian lipid kinase profiling of Torin 2 has been extensively studied and revealed that in addition to mTOR, phosphatidylinositol-3 kinase-like kinase (PIKK) family kinases are major Torin 2 interacting proteins $s^{31,32}$. During the preparation of this manuscript, another group reported that Torin 2 inhibited $P$. falciparum development in the liver stage $\left(\mathrm{IC}_{50}=\right.$ $1.1 \mathrm{nM})$, as well as the asexual blood stage $\left(\mathrm{IC}_{50}=1.4 \mathrm{nM}\right)$ and the early stages (I and II) of P. falciparum gametocytes $\left(\mathrm{IC}_{50}=\right.$ $6.6 \mathrm{nM})^{51}$. In this study, we found that Torin 2 potently killed the gametocyte stages III-V with nanomolar activity and completely blocked gametocyte transmission to mosquitoes in vivo using the mouse model. This work complements the recent report on the effects of Torin 2 on the liver and asexual stages and early gametocytes ${ }^{50}$. Together, the data demonstrate that Torin 2 is effective against sexual and asexual blood stages as well as liver stages of malaria parasites with low nanomolar potency and blocks transmission to mosquitoes at a tolerable dose and thus is an ideal lead compound for the next generation antimalarial agent.
Though Torin 2 was able to prevent oocyst formation in mosquitoes that fed on infected mice, the precise stage of the life cycle affected is unknown. In the $P$. berghei mouse model, parasite exflagellation in the drug-treated animals was equivalent to that of the vehicle-treated controls. These data indicate that the male $P$. berghei gametocytes are still viable $1.5 \mathrm{hrs}$ after drug treatment. It is possible that some amount of Torin 2 carried over into the mosquitoes and affected one of the stages between the gametocyte and the oocyst: gamete, zygote or ookinete. Alternatively, Torin 2 could be affecting a parasite pathway that allows exflagellation, but blocks subsequent steps that are necessary for productive mosquito infection. In either case, Torin 2 remained active in vivo and effectively blocked oocyst production. Future studies will examine which of these stages are affected by Torin 2 using $P$. berghei ookinete cultures.

An mTOR homolog has not been identified in P. falciparum, and the homologies between parasite PIKK and mammalian PIKK are not striking. There is only $22 \%$ identity in the 148 amino acid catalytic domain of PfPI3K and HsPI3 $3 \mathrm{~K}^{33}$. To identify the target of Torin 2 in P. falciparum, we have employed two independent approaches, Torin 2 affinity precipitation and DARTS. Phosphoribosylpyrophosphate synthetase (RPPK), aspartate carbamoyltransferase (ATCase), and a putative transporter PF3D7_0914700 were found in both experiments as the potential targets of Torin 2. RPPK is a kinase that catalyzes the synthesis of PRPP and adenosine monophosphate (AMP) from ribose 5-phosphate and adenosine 5 '-triphosphate $(\mathrm{ATP})^{51}$. Although its activity has not been directly tested in P. falciparum, this enzyme links carbon and nitrogen metabolism and is essential for the salvage and synthesis of purines as well as the synthesis of pyrimidines and pyridines in many species from yeast to humans ${ }^{52}$. It also plays a role in histidine and tryptophan metabolism in bacteria and fungi ${ }^{53}$. In contrast, ATCase catalyzes the first step in the pyrimidine biosynthetic pathway ${ }^{54}$. The participation of these two enzymes in pyrimidine synthesis suggests that the very early steps in this pathway are important for gametocyte growth and survival, which have not been previously recognized as potential targets for gametocytocidal drug development. The third potential target is predicted to be a transporter, which may involve the transfer of charged molecules across cell membrane lipid bilayers. These results open a door to further study gametocyte targets, allowing the understanding the mechanism of action for Torin 2 and accelerating the further development of this compound. Similarly, serotonin antagonists were found as potent gametocytocidal compounds although there is no $P$. falciparum homolog of serotonin receptors, indicative of the existence of a potential gametocyte specific target.

In summary, the cheminformatic analysis of chemical signatures of the active compounds identified from this study against the sexual stages (gametocytes) and asexual blood stages of $P$. falciparum (previously reported) have revealed new chemical signatures and protein targets for future antimalarial drug development. Among 27 newly identified compounds, Torin 2 possesses nanomolar potencies against both asexual and sexual $P$. falciparum parasites with the ability to completely block gametocyte transmission from host to mosquitoes. In addition, RPPK, ATCase, and transporter (PF3D7_0914700) were identified as potential targets of Torin 2. Therefore, this study provides critical new leads and novel targets to accelerate gametocytocidal drug development. We also think that this approach can be extended to other pathogens including bacteria, fungi, protozoa and parasites for rapid identification of new drugs and drug targets.

\section{Methods}

Cell culture. Asexual parasites of $P$. falciparum strain 3D7 were cultured as described previously ${ }^{55}$. Briefly, gametocyte cultures were set up at $0.1 \%$ parasitemia and on days 9-10 treated with $50 \mathrm{mM} \mathrm{N}$-acetylglucosamine (NAG) to block further asexual growth. Stage III-V gametocytes were isolated by Percoll density gradient centrifugation on day 12 and returned to culture for 24 hrs before being used in the assay ${ }^{9}$. At the time of the assay $73 \%$ of the gametocytes were stage IV or V. 
Gametocytes of HB3 and Dd2 strains were produced and then set up for assay in a similar process. HepG2 cells (ATCC, cat. no. 77400 ) were cultured in $175-\mathrm{cm}^{2}$ tissue culture flasks with $30 \mathrm{ml}$ growth medium at $37^{\circ} \mathrm{C}$ in a $5 \% \mathrm{CO}_{2} \& 5 \% \mathrm{O}_{2}$ humidified atmosphere. Growth medium was made with Dulbecco's Modified Eagle Medium with $10 \%$ fetal bovine serum (FBS). Growth medium was replaced every other day and cells were passed at $75 \%$ confluence.

Compound library and gametocyte assay screen. The National Institutes of Health (NIH) Chemical Genomics Center Pharmaceutical Collection (NPC) was constructed in-house through a combination source of traditional chemical suppliers, specialty collections, pharmacies and custom synthesis ${ }^{12}$. Briefly, the NPC library comprises 4,265 small-molecule compounds, $49 \%$ of which are drugs approved for human or animal use by the US Food and Drug Administration (FDA), 23\% are drugs approved in Canada/UK/EU/Japan, and the remaining $28 \%$ are compounds that have entered clinical trials or are research compounds commonly used in biomedical research. The Malaria Box contains 400 drugs or tool compounds that have confirmed activity on blood-staged P. falciparum and assessed cytotoxicity against mammalian cells ${ }^{40,56}$. The MIPE library is an internal collection of 550 kinase inhibitors, which contain approved drugs and compounds in clinical and preclinical stages $^{14}$. Compounds from all libraries were obtained as powder samples and dissolved in DMSO as $10 \mathrm{mM}$ stock solutions, except several hundred from the NPC library that were prepared as $4.47 \mathrm{mM}$ stock solutions due to solubility limitations.

Compound screening experiments were performed as previously described ${ }^{11}$ Briefly, $2.5 \mu \mathrm{l} /$ well incomplete medium was dispensed into each well of 1,536-well plates using the Multidrop Combi followed by $23 \mathrm{nl}$ compound transferring using the NX-TR Pintool (WAKO Scientific Solutions, San Diego, CA). Then, $2.5 \mu \mathrm{l} /$ well of gametocytes was dispensed with a seeding density of 20,000 cells/well using the Multidrop Combi. The assay plates were incubated for $72 \mathrm{hrs}$ at $37^{\circ} \mathrm{C}$ with $5 \% \mathrm{CO}_{2}$. After addition of $5 \mu \mathrm{l} /$ well of $2 \times$ AlamarBlue dye (Life Technologies, cat. no. DAL1100), the plates were incubated for $24 \mathrm{hrs}$ at $37^{\circ} \mathrm{C}$ with $5 \% \mathrm{CO}_{2}$ and then were read in a fluorescence detection mode $(\mathrm{Ex}=525 \mathrm{~nm}, \mathrm{Em}=598 \mathrm{~nm})$ on a ViewLux plate reader (PerkinElmer).

Small molecule pull-down. Affinity matrix: To make a bead-connected affinity probe of Torin 2, a tetraethylene glycol linker was attached to 1-(piperazin-1-yl)propan-1one of HWW030 and then coupled to Affi-Gel 10 resin (Bio-Rad Laboratories, cat. no. 153-6046) under mild basic conditions to afford Torin 2 matrix (T2M). See detailed version in SI methods. Torin 1 was similarly immobilized to resin and used as a negative control (T1M). The resultant affinity probes were incubated with gametocyte lysates, the bound proteins were eluted from resin by boiling in SDSPAGE sample loading buffer. The eluted fractions were separated by SDS-PAGE and visualized by silver staining. RBC infected with gametocytes (3D7 strain: Stages III-V) were washed 3 times with PBS and then lysed by $0.05 \%$ saponin treatment in PBS for 5 mins at room temperature. The prepared gametocytes were washed 3 times with $\mathrm{PBS}$ and frozen at minus $80^{\circ} \mathrm{C}$. The affinity precipitation experiment was processed as previously described ${ }^{35}$. The frozen samples were lysed with homogenization buffer (60 mM glycerophosphate, $15 \mathrm{mM}$ p-nitrophenyl phosphate, $25 \mathrm{mM}$ MOPS (pH 7.2), $15 \mathrm{mM}$ EGTA, $15 \mathrm{mM} \mathrm{MgCl}$, $1 \mathrm{mM}$ DTT, protease inhibitors (Roche Diagnostics, cat. no. 11836170001), and 0.5\% Nonidet P-40). Cell lysates were centrifuged at $16,000 \times \mathrm{g}$ for $20 \mathrm{mins}$ at $4^{\circ} \mathrm{C}$, and the supernatant was collected. Protein concentration in the supernatant was determined by using a BCA protein assay kit (Pierce Chemical, cat. no. 23225). The lysate $(0.5 \mathrm{mg})$ was then added to the packed affinity matrix, and bead buffer ( $50 \mathrm{mM}$ Tris $\mathrm{HCl}(\mathrm{pH} 7.4), 5 \mathrm{mM} \mathrm{NaF}$, $250 \mathrm{mM} \mathrm{NaCl}, 5 \mathrm{mM}$ EDTA, $5 \mathrm{mM}$ EGTA, protease inhibitors, and $0.1 \%$ Nonidet P40) was added to a final volume of $1 \mathrm{ml}$. After rotating at $4^{\circ} \mathrm{C}$ for $2 \mathrm{hrs}$, the mixture was centrifuged at $16,000 \times g$ for 2 mins at $4^{\circ} \mathrm{C}$, and the supernatant was removed. The affinity matrix was then washed (six times) with cold bead buffer and eluted by boiling with SDS-PAGE sample loading buffer at $95^{\circ} \mathrm{C}$ for 5 mins. Supernatants were separated on a 10\% Bis-Tris gel (Life Technologies) and visualized by silver staining using a Pierce Silver Stain Kit for Mass Spectrometry (Pierce Chemical).

DARTS. The 3D7 gametocytes were lysed with M-PER (Peirce Chemical) supplemented with protease and phosphatase inhibitors as previously described ${ }^{10}$. After centrifugation at $16,000 \times g$ for 20 mins, protein concentration in the supernatant was quantified and $2 \mu \mathrm{g} / \mu \mathrm{l}$ proteins were treated with $600 \mathrm{nM}$ of Torin 2 or $600 \mathrm{nM}$ of Torin 1 for $2 \mathrm{hrs}$ at room temperature. The samples were treated with $46 \mu \mathrm{g} / \mathrm{ml}$ pronase (Sigma-Aldrich, cat. no. P6911) for $30 \mathrm{mins}$ at room temperature. The digestion was stopped by adding the SDS-PAGE sample loading buffer and boiled at $70^{\circ} \mathrm{C}$ for 10 mins. The samples were separated on a $10 \%$ Bis-Tris gel and visualized by silver staining.

Mouse malaria model. Plasmodium berghei ANKA $(\mathrm{Pb})$ parasites were maintained by serial passage by intraperitoneal (i.p.) injection in outbred mice. All parasites used were serially passaged less than 5 times. Two days before feeding, female mice were infected i.p. with $200-400 \mu \mathrm{l}$ whole blood from a Pb-infected mouse with $>10 \%$ parasitemia, resulting in a high parasitemia in a short, predictable amount of time. On day 2 post infection, the mice were checked for exflagellation. If exflagellation was not observed, it was reassessed on day 3 . All mice exhibited exflagellation on either day 2 or day 3. After exflagellation was observed, the mice were treated intravenously (i.v.) with drug vehicle alone ( $10 \% \mathrm{~N}$-methylpyrrolidnone, $40 \%$ PEG 400 in water) or $2-4 \mathrm{mg} / \mathrm{kg}$ Torin 2 . Mice either received two doses $3 \mathrm{hrs}$ apart or a single dose. The two dose treatment was done in triplicate, and the one dose treatment was done once.
1.5 hours after the final treatment, mice were anesthetized and 20-30 female Anopheles stephensi mosquitoes (6-9 days old) were allowed to feed on an infected mouse for 15 mins. Parasitemia, gametocytemia, and presence of exflagellation were examined as described previously ${ }^{57}$. Mosquitoes were maintained on $5 \%(w / v)$ glucose at $19^{\circ} \mathrm{C}$ and $80 \%$ relative humidity. At day 10 post feeding, mosquito midguts were dissected and parasite infection was measured by staining mosquito midguts with $0.2 \%$ mercurochrome and counting the numbers of oocysts per midgut. Statistical significance was determined using Student's T-test when comparing two treatment groups and One-Way ANOVA when comparing three groups. All animal experiments were done at Loyola University Chicago, in compliance with the guidelines of their Institutional Animal Care and Use Committee.

Data analysis. The primary screen data was analyzed using customized software developed internally ${ }^{58}$. IC $_{50}$ values were calculated using the Prism software (Graphpad Software, Inc. San Diego, CA). Data were presented as means \pm SEM with $\mathrm{n}=3$ independent experiments.

1. Kilama, W. \& Ntoumi, F. Malaria: a research agenda for the eradication era. Lancet 374, 1480-1482, doi:10.1016/S0140-6736(09)61884-5 (2009).

2. Buchholz, K. et al. A high-throughput screen targeting malaria transmission stages opens new avenues for drug development. J Infect Dis 203, 1445-1453, doi:10.1093/infdis/jir037 (2011).

3. Sweeney, A. W., Blackburn, C. R. B. \& Rieckmann, K. H. Short report: The activity of pamaquine, an 8-aminoquinoline drug, against sporozoite-induced infections of Plasmodium vivax (New Guinea strains). Am J Trop Med Hyg 71, 187-189 (2004).

4. Peatey, C. L. et al. Effect of Antimalarial Drugs on Plasmodium falciparum Gametocytes. Journal of Infectious Diseases 200, 1518-1521, doi:Doi 10.1086/ 644645 (2009).

5. Baird, J. K. \& Surjadjaja, C. Consideration of ethics in primaquine therapy against malaria transmission. Trends Parasitol 27, 11-16, doi:Doi 10.1016/ J.Pt.2010.08.005 (2011).

6. Shekalaghe, S. A. et al. A cluster-randomized trial of mass drug administration with a gametocytocidal drug combination to interrupt malaria transmission in a low endemic area in Tanzania. Malaria J 10, doi:Artn 247 Doi 10.1186/14752875-10-247 (2011)

7. Coulibaly, B. et al. Strong gametocytocidal effect of methylene blue-based combination therapy against falciparum malaria: a randomised controlled trial. PLoS One 4, e5318, doi:10.1371/journal.pone.0005318 (2009).

8. Peatey, C. L., Spicer, T. P., Hodder, P. S., Trenholme, K. R. \& Gardiner, D. L. A high-throughput assay for the identification of drugs against late-stage Plasmodium falciparum gametocytes. Mol Biochem Parasitol 180, 127-131, doi:10.1016/j.molbiopara.2011.09.002 (2011).

9. Tanaka, T. Q. \& Williamson, K. C. A malaria gametocytocidal assay using oxidoreduction indicator, alamarBlue. Mol Biochem Parasitol 177, 160-163, doi:DOI 10.1016/j.molbiopara.2011.02.005 (2011).

10. Lomenick, B. et al. Target identification using drug affinity responsive target stability (DARTS). Proc Natl Acad Sci U S A 106, 21984-21989, doi:DOI 10.1073/ pnas.0910040106 (2009).

11. Tanaka, T. Q. et al. A quantitative high throughput assay for identifying gametocytocidal compounds. Mol Biochem Parasitol 188, 20-25, doi:10.1016/ j.molbiopara.2013.02.005 (2013).

12. Huang, R. et al. The NCGC pharmaceutical collection: a comprehensive resource of clinically approved drugs enabling repurposing and chemical genomics. Sci Transl Med 3, 80ps16, doi:10.1126/scitranslmed.3001862 (2011).

13. Duffy, S. \& Avery, V. M. Development and Optimization of a Novel 384-Well Anti-Malarial Imaging Assay Validated for High-Throughput Screening. Am J Trop Med Hyg 86, 84-92, doi:DOI 10.4269/ajtmh.2012.11-0302 (2012).

14. Mathews, L. A. et al. A 1536-Well Quantitative High-Throughput Screen to Identify Compounds Targeting Cancer Stem Cells. J Biomol Screen 17, 1231-1242, doi:Doi 10.1177/1087057112458152 (2012).

15. Benoit-Vical, F. et al. Trioxaquines are new antimalarial agents active on all erythrocytic forms, including gametocytes. Antimicrob Agents Chemother 51, 1463-1472, doi:Doi 10.1128/Aac.00967-06 (2007).

16. Lelievre, J. et al. Activity of Clinically Relevant Antimalarial Drugs on Plasmodium falciparum Mature Gametocytes in an ATP Bioluminescence “Transmission Blocking" Assay. PLoS One 7, doi:ARTN e35019 DOI 10.1371/ journal.pone.0035019 (2012).

17. Yuan, J. et al. Chemical genomic profiling for antimalarial therapies, response signatures, and molecular targets. Science 333, 724-729, doi:10.1126/ science.1205216 (2011)

18. Yuan, J. et al. Genetic mapping of targets mediating differential chemical phenotypes in Plasmodium falciparum. Nat Chem Biol 5, 765-771, doi:10.1038/ nchembio.215 (2009).

19. Kohonen, T. Self-organizing neural projections. Neural Netw 19, 723-733, doi:10.1016/j.neunet.2006.05.001 (2006)

20. Tin, F. \& Nyunt, H. Comparative drug trial of a sulfadoxine/pyrimethamine and a sulfalene/pyrimethamine combination against Plasmodium falciparum infections in semi-immune populations of Burma. Southeast Asian J Trop Med Public Health $15,238-248(1984)$ 
21. Butcher, G. A. Antimalarial drugs and the mosquito transmission of Plasmodium. Int J Parasitol 27, 975-987 (1997).

22. Reininger, L., Wilkes, J. M., Bourgade, H., Miranda-Saavedra, D. \& Doerig, C. An essential Aurora-related kinase transiently associates with spindle pole bodies during Plasmodium falciparum erythrocytic schizogony. Mol Microbiol 79, 205-221, doi:10.1111/j.1365-2958.2010.07442.x (2011).

23. Andrews, K. T. et al. Comparative gene expression profiling of P. falciparum malaria parasites exposed to three different histone deacetylase inhibitors. PLoS One 7, e31847, doi:10.1371/journal.pone.0031847 (2012).

24. Lucantoni, L. \& Avery, V. Whole-cell in vitro screening for gametocytocidal compounds. Future Med Chem 4, 2337-2360, doi:10.4155/fmc.12.188 (2012).

25. Chen, P. Q. et al. The infectivity of gametocytes of Plasmodium falciparum from patients treated with artemisinin. Chin Med J (Engl) 107, 709-711 (1994).

26. Bousema, J. T. et al. Moderate effect of artemisinin-based combination therapy on transmission of Plasmodium falciparum. J Infect Dis 193, 1151-1159, doi:10.1086/ 503051 (2006).

27. Fidock, D. A., Rosenthal, P. J., Croft, S. L., Brun, R. \& Nwaka, S. Antimalarial drug discovery: efficacy models for compound screening. Nat Rev Drug Discov 3, 509-520, doi:10.1038/nrd1416 (2004).

28. Kidgell, C. et al. A systematic map of genetic variation in Plasmodium falciparum. PLoS Pathog 2, e57, doi:10.1371/journal.ppat.0020057 (2006).

29. Baniecki, M. L., Wirth, D. F. \& Clardy, J. High-throughput Plasmodium falciparum growth assay for malaria drug discovery. Antimicrob Agents Chemother 51, 716-723, doi:10.1128/AAC.01144-06 (2007).

30. Lai, C. J. et al. CUDC-101, a multitargeted inhibitor of histone deacetylase, epidermal growth factor receptor, and human epidermal growth factor receptor 2 , exerts potent anticancer activity. Cancer Res 70, 3647-3656, doi:10.1158/00085472.CAN-09-3360 (2010).

31. Liu, Q. et al. Characterization of Torin2, an ATP-Competitive Inhibitor of mTOR, ATM, and ATR. Cancer Res 73, 2574-2586, doi:10.1158/0008-5472.CAN-12$1702(2013)$.

32. Liu, Q. et al. Discovery of 9-(6-aminopyridin-3-yl)-1-(3(trifluoromethyl)phenyl)benzo[h][1,6]naphthyridin-2(1H)-one (Torin2) as a potent, selective, and orally available mammalian target of rapamycin (mTOR) inhibitor for treatment of cancer. J Med Chem 54, 1473-1480, doi:10.1021/ jm101520v (2011).

33. Bharatham, N., Chang, M. W. \& Yoon, H. S. Targeting FK506 Binding Proteins to Fight Malarial and Bacterial Infections: Current Advances and Future Perspectives. Curr Med Chem 18, 1874-1889 (2011).

34. Coleman, R. E., Clavin, A. M. \& Milhous, W. K. Gametocytocidal and Sporontocidal Activity of Antimalarials against Plasmodium-Berghei Anka in Icr Mice and Anopheles-Stephensi Mosquitos. Am J Trop Med Hyg 46, 169-182 (1992).

35. Zhang, Q. et al. Small-molecule synergist of the Wnt/beta-catenin signaling pathway. Proc Natl Acad Sci US A 104, 7444-7448, doi:10.1073/pnas.0702136104 (2007).

36. Purcell, W. P. Some Guidelines for Application of Quantitative StructureActivity-Relationships in Drug Design. Eur J Med Chem 10, 335-339 (1975).

37. Dechy-Cabaret, O. \& Benoit-Vical, F. Effects of antimalarial molecules on the gametocyte stage of Plasmodium falciparum: the debate. J Med Chem 55, 10328-10344, doi:10.1021/jm3005898 (2012).

38. Toye, P. J., Sinden, R. E. \& Canning, E. U. The action of metabolic inhibitors on microgametogenesis in Plasmodium yoelii nigeriensis. Z Parasitenkd 53, 133-141 (1977).

39. Gamo, F. J. et al. Thousands of chemical starting points for antimalarial lead identification. Nature 465, 305-U356, doi:Doi 10.1038/Nature09107 (2010).

40. Plouffe, D. et al. In silico activity profiling reveals the mechanism of action of antimalarials discovered in a high-throughput screen. Proc Natl Acad Sci U S A 105, 9059-9064, doi:DOI 10.1073/pnas.0802982105 (2008).

41. Derbyshire, E. R., Prudencio, M., Mota, M. M. \& Clardy, J. Liver-stage malaria parasites vulnerable to diverse chemical scaffolds. Proc Natl Acad Sci U S A 109, 8511-8516, doi:DOI 10.1073/pnas.1118370109 (2012).

42. Hersey, P. et al. Small molecules and targeted therapies in distant metastatic disease. Ann Oncol 20, 35-40, doi:DOI 10.1093/annonc/mdp254 (2009).

43. Eccles, S. A. et al. NVP-AUY922: A novel heat shock protein 90 inhibitor active against xenograft tumor growth, angiogenesis, and metastasis. Cancer Res $\mathbf{6 8}$, 2850-2860, doi:Doi 10.1158/0008-5472.Can-07-5256 (2008)

44. Sessa, C. et al. First-in-human phase I dose-escalation study of the HSP90 inhibitor AUY922 in patients with advanced solid tumors. Clin Cancer Res 19, 3671-3680, doi:10.1158/1078-0432.CCR-12-3404 (2013).
45. Pacey, S. et al. A phase I study of the heat shock protein 90 inhibitor alvespimycin (17-DMAG) given intravenously to patients with advanced solid tumors. Clin Cancer Res 17, 1561-1570, doi:10.1158/1078-0432.CCR-10-1927 (2011).

46. Banumathy, G., Singh, V., Pavithra, S. R. \& Tatu, U. Heat shock protein 90 function is essential for Plasmodium falciparum growth in human erythrocytes. $J$ Biol Chem 278, 18336-18345, doi:10.1074/jbc.M211309200 (2003).

47. Kumar, R., Musiyenko, A. \& Barik, S. The heat shock protein 90 of Plasmodium falciparum and antimalarial activity of its inhibitor, geldanamycin. Malaria J $\mathbf{2}$, 30, doi:10.1186/1475-2875-2-30 (2003).

48. Pesce, E. R., Cockburn, I. L., Goble, J. L., Stephens, L. L. \& Blatch, G. L. Malaria heat shock proteins: drug targets that chaperone other drug targets. Infect Disord Drug Targets 10, 147-157 (2010).

49. Singh, C. \& Atri, N. Chemo-informatic design of antibiotic geldenamycin analogs to target stress proteins HSP90 of pathogenic protozoan parasites. Bioinformation 9, 329-333, doi:10.6026/97320630009329 (2013).

50. Hanson, K. K. et al. Torins are potent antimalarials that block replenishment of Plasmodium liver stage parasitophorous vacuole membrane proteins. Proc Natl Acad Sci U S A 110, E2838-E2847, doi:DOI 10.1073/pnas.1306097110 (2013).

51. Reyes, P. et al. Enzymes of purine and pyrimidine metabolism from the human malaria parasite, Plasmodium falciparum. Mol Biochem Parasitol 5, 275-290 (1982).

52. Varvassori, S., Wang, K., Schweizer, L. M. \& Schweizer, M. Ramifications of impaired PRPP synthesis in Saccharomyces cerevisiae. Biochem Soc T 33 1418-1420 (2005).

53. Hovejensen, B. Phosphoribosylpyrophosphate (Prpp)-Less Mutants of Escherichia-Coli. Mol Microbiol 3, 1487-1492, doi:DOI 10.1111/j.13652958.1989.tb00134.x (1989).

54. Tampitag, S. \& O'Sullivan, W. J. Enzymes of pyrimidine biosynthesis in Crithidia luciliae. Mol Biochem Parasitol 19, 125-134 (1986).

55. Trager, W. \& Jensen, J. B. Human malaria parasites in continuous culture. 1976. J Parasitol 91, 484-486, doi:10.1645/0022-3395(2005)091[0484:HMPICC] 2.0.CO;2 (2005).

56. Guiguemde, W. A. et al. Chemical genetics of Plasmodium falciparum. Nature 465, 311-315, doi:Doi 10.1038/Nature09099 (2010).

57. Blagborough, A. M. et al. Transmission-blocking interventions eliminate malaria from laboratory populations. Nat Commun 4, 1812, doi:10.1038/ncomms2840 (2013).

58. Wang, Y., Jadhav, A., Southal, N., Huang, R. \& Nguyen, D. T. A grid algorithm for high throughput fitting of dose-response curve data. Curr Chem Genomics 4, 57-66, doi:10.2174/1875397301004010057 (2010).

\section{Acknowledgments}

This work was supported by the Intramural Research Programs of the National Center for Advancing Translational Sciences and National Institute of Allergy and Infectious Diseases, and Public Health Service grant AI101396 from the National Institute of Allergy and Infectious Diseases. TQT is a JSPS Research Fellow in Biomedical and Behavioral Research at the NIH. We thank Mr. Paul Shinn from the National Center for Advancing Translational Sciences for preparing compound plates and Ms. Sullivan from the National Institute of Allergy and Infectious Diseases for technical assistance. We also thank Dr. Zhaojing Meng from SAIC for mass spectrometry analysis.

\section{Author contributions}

K.C.W., W.Z., T.Q.T. and W.S. designed the study. W.S., T.Q.T., C.T.M., W.W.H., R.H. and D.J.S. performed the experiments, and collected and analyzed the data. W.S., T.Q.T., R.H., K.C.W. and W.Z. wrote the manuscript. N.S., R.H. and C.T.M. performed statistical analysis. J.C.M. aided study design. All authors discussed the results and commented on the manuscript.

\section{Additional information}

Supplementary information accompanies this paper at http://www.nature.com/ scientificreports

Competing financial interests: The authors declare no competing financial interests. How to cite this article: Sun, W. et al. Chemical signatures and new drug targets for gametocytocidal drug development. Sci. Rep. 4, 3743; DOI:10.1038/srep03743 (2014).

This work is licensed under a Creative Commons Attribution 3.0 Unported license. To view a copy of this license, visit http://creativecommons.org/licenses/by/3.0 